\title{
Evaluating Multiple Antenna Diversity Techniques for Transmission over Fading Channels
}

\author{
Ekwe, O.A ${ }^{1}$, Anorue E. N. ${ }^{2}$, Okoro, C.K ${ }^{3}$ \\ ${ }^{123}$ (Department of Electrical/Electronics, Michael Okpara University of Agriculture, Umudike).
}

\begin{abstract}
Diversity is a powerful technique to mitigate fading, hence multiple antenna diversity which includes; multiple devices, spatial diversity, cooperative diversity which uses multiple-element transmitter arrays enhance performance greatly in modern wireless communication networks. Appropriate combining at the receiver realizes diversity gain. major interest in this write up is the MIMO \{Multiple-input and Multipleoutput $\}$ antenna method, it is one of the best forms of smart antenna technology and Dynamic control processes for reducing fading over channels by multiple antenna diversity techniques
\end{abstract}

Keywords: Antenna Arrays, Fading channels, Diversity techniques, Transmission, Fading channel Feedback communication, Wireless communication, Channel coding

\section{Introduction}

Multiple-element transmitter antenna arrays have an increasingly important role to play in emerging wireless communication networks, particularly at base stations in cellular systems. When used in conjunction with appropriately designed signal processing algorithms, such arrays can dramatically enhance performance. Transmitter arrays have long been used for beam forming in radio communications. The potential for using such arrays in switched diversity scheme is similar to those used in receiver arrays.

Transmitter arrays may be used in point - to - point scenarios in which the transmitters have no knowledge of the channel parameters, or equivalently in broadcast scenarios where there are infinitely many recipients.

Transmission over wireless channels suffers from random fluctuations in signal level known as fading and co-channel interference.

Diversity is a powerful technique to mitigate fading. [1]

\section{Background Review}

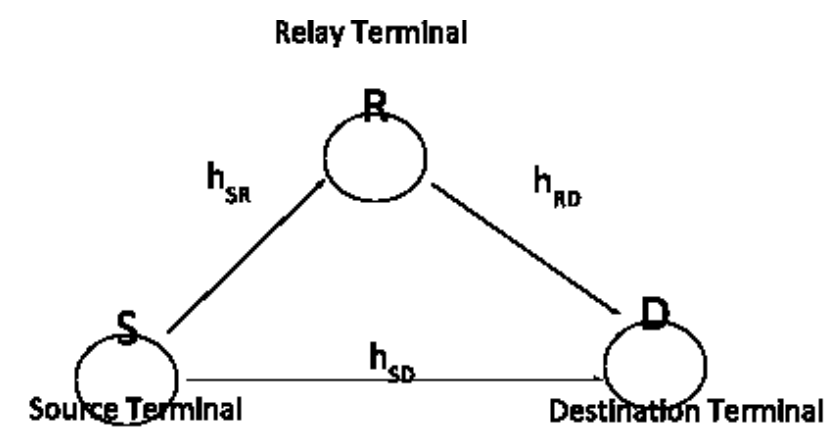

Fig.1.Schematic of fading relay channel

Diversity improves robustness to interference. In classical diversity techniques, the data signal is converged to the receiver over multiple (ideally) independently fading signal paths (in time/frequency/space). Appropriate combining at the receiver realizes diversity gain, thereby improving link reliability.

Spatial or antenna diversity techniques are particularly attractive since they provide diversity gain without incurring an expenditure of transmission time or bandwidth. Signal design for multi-antenna systems with collocated antennas (also known as space-time coding) aimed at extracting spatial diversity.

A new way of reading spatial diversity gain (in a distributed fashion) has recently been introduced under the name of user cooperation diversity or cooperative diversity.

Here, multiple terminals (sensors) in a network cooperate to form a virtual antenna array realizing spatial diversity in a distributed fashion. 
It has been demonstrated that uplink capacity can be increased via user cooperation diversity. A variety of cooperation protocols for channels with a single relay terminal have been studied and analyzed. It is shown that for channels with multiple relays, cooperative diversity with appropriately designed codes realizes full spatial diversity gain.

We may note that many cooperative diversity schemes can be cast into the framework of network coding (e.g. CDMA).

\section{Theory}

Understanding of SISO, SIMO, MIMO

(Note that the terms input and output refers the radio channel carrying the signal; not to the devices having antenna).

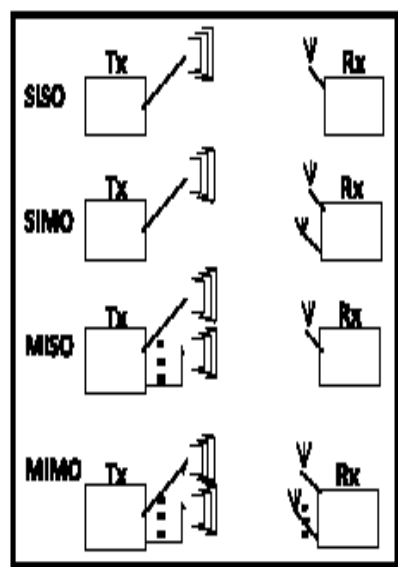

Fig .2 IV.ANALYSING MULTIPLE ANTENNAS.

Multiple Antennas are devices used at both the transmitter and receiver to improve communication performance. Hence, it is commonly used in radio as MIMO, multiple-Input and Multiple Output. It is one of several forms of smart antenna technology.

MIMO technology has attracted attention in wireless communication, because it offers significant increases in data throughput and link range without additional bandwidth or increased power. It achieves this goal by spreading the same total transmit power over the antennas to achieve array gain that improve the spectral efficiency (more bits per second per hertz of bandwidth) or to achieve a diversity gain that improves the link reliability (reduced fading). Because of these properties, MIMO is an important part of modern wireless communication standard such as IEEE 802.11n (Wi Fi), 4G, 3GPP Long term evolution, W.Max and HSPA+

\subsection{Diversity techniques}

Providing architecture with diversity is important for maintaining high performance in wireless mobile communications.

Diversity can be achieved by using Multiple antennas, using interleaved coded modulation, resolving propagation paths in time or spatially and using multicarrier transmission. [2],[3]. Perhaps the most commonly used technique is interleaved coded modulation.

The coding adds the redundancy to provide diversity and the interleaving separates the code symbols to (hopefully) provide independent fading distortion for each of the code symbols. The problem with standard interleaved coded modulation is that a tradeoff must be made between decoding delay (a function of the interleave depth) and demodulation performance. This is especially important in applications where performance is decoding delay sensitive (e.g. voice transmission). For situations with small Doppler spread (e.g., pedestrian or stopped vehicle) either a very long interleaver is needed to achieve quasa-independent distortion on code symbols or else interleaving is not effective.

An effective technique in wireless communication in transmission diversity is that by transmitting from multiple spatially separated antennas (e.g. a base station). Diversity can be achieved without greatly increasing the complexity of the receiver. (E.g. a portable unit). [1]

The simplest ideal is to switch between the transmitters at different time instantly and allow only one transmitter to be on at a time. Because the transmitters are operating intermittently, their peak power is considerably higher than their average power, which complicates the outputs of their output amplifiers.

Other transmission diversity techniques that do not switch off the transmitters are ones using an intentional time offset or frequency offset, phase sweeping, frequency hopping and modulation diversity.

Most of these techniques use phase or frequency modulation of each transmitter carrier to induce intentional time-varying fading at the receiver. The advantage of this technique is that the modulation level of the carrier and the interlacing depth can be chosen to achieve near ideal interleaving. In these applications, a shorter 
interleave depth is usually only achieved with an expanded signal bandwidth.

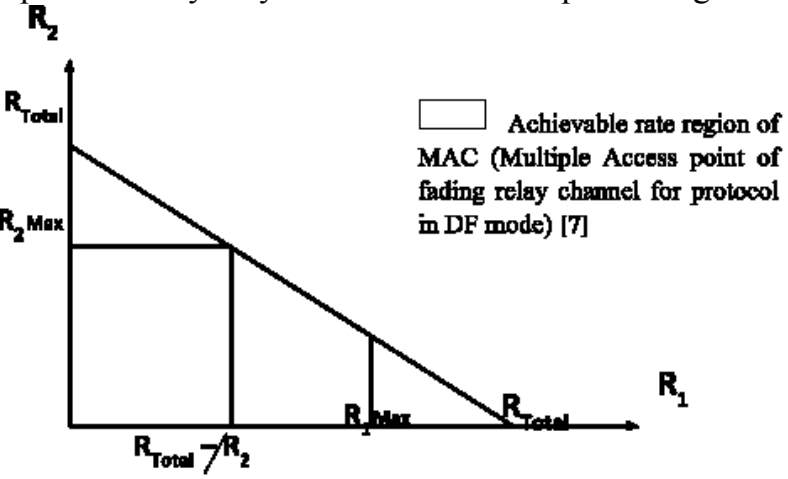

Fig.3

\subsection{Fading Channels} of AWGN

Over large distances; signal quality is shown to degrade eve $\mathrm{n}$ without the presence of large quantities channels [4]

This degradation is known as fading and channels that exhibit these properties are known as fading

\subsection{Causes of Fading}

Natural weather disturbances; such as rainfall, snows, fog, bail and extremely cold air over a warm earth.

Man-made disturbances such as; irrigation from multiple transmission paths, irregular earth surfaces, and varying terrains.

\subsection{Fading Channel Modes}

$>$ Rician fading

$>$ Rayleigh fading

$>$ Nagami fading

\subsection{Scales of Fading}

$>$ Large-scale fading

$>$ Small scale fading

$>$ Frequency-selective fading

$>$ Flat fading

$>$ Fast fading.

SENDER

PROPAGATION RiECEVER

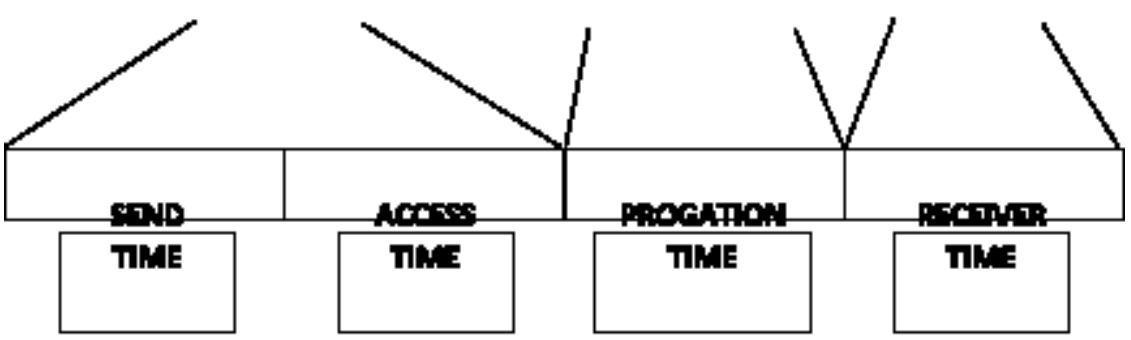

Fig .4 


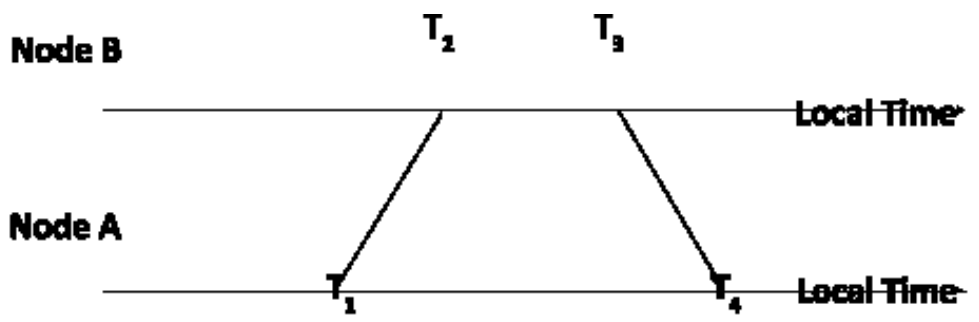

Fig .5

[8]

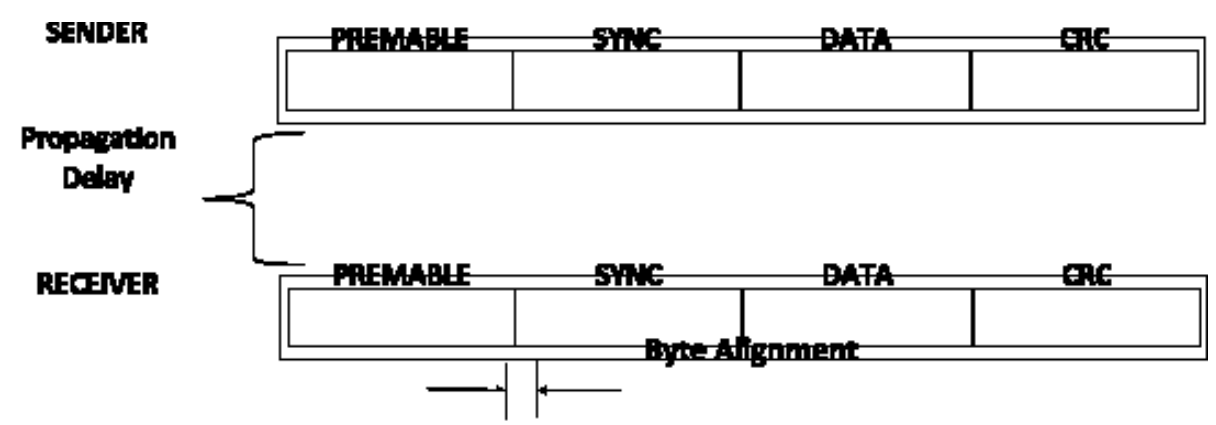

Fig .6

[9]

\subsection{Antenna Techniques.}

Antenna diversity can be realised in several ways depending on the environment and the expected interference. Multiple methods are frequently used to further increase reliability.

Spatial diversity - Cellularization (have base stations miles apart).

Pattern diversity -2 or more co-located antennas with different radiation patterns.

Polarization diversity - Combines pairs of antenna useful on radio mobile communication.

Transmit/receive diversity - Uses two separate co-located antennas for transmitting and receiving.

Adaptive arrays - Can be a single antenna with active elements or an array of similar antenna with ability to change their combined radiation.

\section{Result Pprocessing Techniques of Multiple Antenna Diversity Technique for Transmision over Fading Channels.}

All of these techniques require some sort of past processing to recover the desired message, among these techniques are:

SWITCHING > In a switching recovery, the signal from only one antenna is fed to the receiver for as long as the quality of that signal remains above some prescribed threshold. If and when the signal degrades, another antenna is switched in. Switching is the easiest and least power consuming of antenna diversity processing techniques but periods of fading and DE synchronization may occur while the quality of one antenna degrades and another antenna link is established. [5]

SELECTING > as with switching, selection processing presents only one antenna's signal to the receiver at any given time. The antenna chosen however is based on the best signal to noise ratio (SNR) among the required signals. This requires that a pre-measurement take place and that all antenna have established connectors (at least during the SNR measurement) leading to a higher power requirement. The actual selection process can be taken place in-between received packets of information. This ensures that a simple antenna connection is maintained as much as possible switching can take place on a packet by packet basis if necessary.

COMBINING > In combining, all antennas maintain established connections at all times. The signals are then combined and presented to the receiver. Depending on the sophistication of the subjects, the signals can be added directly (equal gain combining) or weighed and added coherently Maximal-Ratio-Combining. Such a system provides the greatest resistances to fading but since all the receive paths must remain energised, it also consumes the most power. 
DYNAMIC CONTROL $>$ dynamically controlled receivers are capable of choosing from the above processing schemes for wherever the situation arises. While much more complex, they optimize the power versus performance trade-off. Transitions between modes and or antenna connections are signalised by a change in the perceived quality link. In situations of low fading, the receiver can employ no diversity and use the signal presented by a single antenna. As conditions degrade the receiver can then assume the more highly reliable power hungry modes described above. [4]

In long term evolution, W.MAX and HSPA +

\section{Applications}

A well known practical application of diversity reception is in Wireless Microphones.

Also in similar electronic devices such as wireless guitar system. A wireless microphone with a non-diversity receiver (a receiver having only one antenna) is prone to random drops-outs, fades, noise or other interferences; especially if the transmitter (the wireless microphone) is in motion. A wireless microphone or sound system using diversity reception will switch to the other antenna within microsecond. If one antenna experience noise, providing an improved quality signal with fewer dropouts and noise.

Ideally, no dropouts or noise will occur in the received signal.

Another common usage is in Wi Fi networking gear and cordless telephones to compensate for multipath interference. The base station will switch reception to one of two antenna depending on which is currently receiving a stronger signal. For best results, the antenna are usually placed one wavelength apart for microwave bands, where the wavelengths, are under $100 \mathrm{~cm}$, this can often be done with two antennas attached to the same hardware. For lower frequencies and longer wavelengths, the antenna must be several meters apart, making it much less reasonable.

Mobile phone towers also often take advantage of diversity, each face (sector) of a tower will often have two antenna, one is transmitting and receiving; while the other is a receivers are used to perform diversity reception. [2]

Cell antenna on an electricity pylon using two antennas per sector. (MIMO) systems.

The use of multiple antennas at both transmits and receive results in multiple-inputs multiple output

The use of diversity techniques at both end of the link is termed Space-time coding.

Wireless channels severally suffer from fading phenomena which causes unreliability in data decoding. Fundamentally diversity coding sends multiple copies through multiple transmit antennas, so as to improve the reliability of the data reception. If one of them fails to receive the others are used for data decoding.

\subsection{Recommendation}

Since long term evolution, W.MAS and HSPA+ all make use of multiple antenna diversity techniques over fading channels. It would be recommended among the processes used that the DYNAMIC CONTROL should be used more for its advantages over other processes.

Amongst to numerous applications, It would be highly encouraged to use the MIMO systems in multiple antenna diversity techniques over fading channels since it is highly reliable if one of them fails to receive the others are used for data decoding.

\section{Conclusion}

It can be thus deduced that while non-diversity receiver are prone to random drop-outs, fades, noise or other interferences. Multiple antenna diversity techniques reduce fading over channels is highly reliable.

\section{References}

[1]. A Sendonan, E. Erkip and B. Hahang. User Cooperation diversity techniques Part 1, System description. IEEE Trans. Commun, Vol 51 pp. $1927-1938$, Nov 2003

[2]. J. Moon and Y. Kim. "Antenna Diversity strength Wireless LANs". Communication systems Design, Pp. 15-22, Jan 2003

[3]. Carl Dietrich, Jr. "Adaptive Arrays and Diversity Antenna Configurations for Handheld Wireless Communication Terminals". Feb 152000

[4]. G.J Foschin "layered space time architecture for wireless communication in a fading environment when using multi-element antenna Bell labs Tech J. Vol. 1 and 2, pp., 41-59 autumns 1996.

[5] A. Naguib, N. Sesshadri, and A. R. Calderbank "Increasing data rate over wireless channels" IEEE signal processing Mag. Pp. 7692 May 2000

[6] "Adaptive Arrays and Diversity Antenna Configurations for Handheld Wireless Communication Terminals" by Carl Dietrich, Jr. Feb 152000

[7] "Adaptive Antenna Tutorial: Spectral Efficiency and Spacial Processing" by Marc Goldberg. FEC office of Engineering and Technology. Sept 7, 2001

[8] C.D. Iskander. "A MAT LAB - based object-oriented Approach to Multipath fading Channel Simulation. Feb 2008.

[9] S. M. Lindenmen, L. M. Rieter, D. E. Barie and T. F. Hopf. "Antenna Diversity for Improving the BER in Mobile Digital Radio Reception especially in Areas with Dense Foliage" International ITG conference on Antennas, ISBN 978-3-00-021643-5 Pages 4548 Mar 302007. 
Engr. EKWE O. A. is a lecturer at Michael Okpara University of Agriculture, Umudike. He obtained his Bachelor of Engineering (B.Eng.) degree in Electronics Engineering at the University of Nigeria, Nsukka in 2005, and a Master's Degree in Electronic Communications and Computer Engineering from University of Nottingham, United Kingdom in 2011. His research interest are in the areas of Interference management for cellular communication, Communication techniques for next generation cellular systems, Channel fading mitigation for fixed and mobile wireless communication systems, etc.

ANORUE, E. N. B. Eng. is also a postgraduate student of electrical/electronics, Michael Okpara University of Agriculture, Umudike, Abia State, Nigeria. She graduated from the same University with a degree in electrical/electronics engineering. Her research areas include: antenna system, error correction codes, etc.

Okoro Kalu Christopher received his B.Eng. degree in Electrical and Electronics Engineering from Madonna University, Nigeria in 2008, a master's degree in Power System Engineering, University of Lagos, Nigeria in 2013.and currently doing a Phd in the department of Electrical and Electronics Engineering, Michael Okpara University of Agriculture, Umudike, Abia State Nigeria. He is a member of Institute of Electrical and Electronics Engineers. Okoro K.C is presently lecturing in the department of Electrical/Electronic Engineering, Michael Okpara University of Agriculture, Umudike, Abia State, Nigeria. His research interests are in the areas of Electrical Machine drives, Power Automation Systems etc. 